\title{
BICEP/Keck constraints on attractor models of inflation and reheating
}

\author{
John Ellis $\odot,{ }_{1}^{1}$ Marcos A. G. Garcia๑, ${ }^{2}$ Dimitri V. Nanopoulos, ${ }^{3}$ Keith A. Olive, ${ }^{4}$ and Sarunas Verner ${ }^{4}$ \\ ${ }^{1}$ Theoretical Particle Physics and Cosmology Group, Department of Physics, King's College London, \\ London WC2R 2LS, United Kingdom; Theoretical Physics Department, CERN, \\ CH-1211 Geneva 23, Switzerland; NICPB, Rävala puiestee 10, 10143 Tallinn, Estonia \\ ${ }^{2}$ Departamento de Física Teórica, Instituto de Física, Universidad Nacional Autónoma de México, \\ A.P. 20-364, Ciudad de México 01000, Mexico \\ ${ }^{3}$ George P. and Cynthia W. Mitchell Institute for Fundamental Physics and Astronomy, \\ Texas A\&M University, College Station, Texas 77843, USA; \\ Astroparticle Physics Group, Houston Advanced Research Center (HARC), \\ Mitchell Campus, Woodlands, Texas 77381, USA; \\ Academy of Athens, Division of Natural Sciences, Athens 10679, Greece \\ ${ }^{4}$ William I. Fine Theoretical Physics Institute, School of Physics and Astronomy, University of Minnesota, \\ Minneapolis, Minnesota 55455, USA
}

(Received 29 December 2021; accepted 14 January 2022; published 3 February 2022)

\begin{abstract}
Recent BICEP/Keck data on the cosmic microwave background, in combination with previous WMAP and Planck data, impose strong new constraints on the tilt in the scalar perturbation spectrum, $n_{s}$, as well as the tensor-to-scalar ratio, $r$. These constrain the number of $e$-folds of inflation, $N_{*}$, the magnitude of the inflaton coupling to matter, $y$, and the reheating temperature, $T_{\text {reh }}$, which we evaluate in attractor models of inflation as formulated in no-scale supergravity. The $68 \%$ C.L. region of $\left(n_{s}, r\right)$ favors large values of $N_{*}, y$, and $T_{\text {reh }}$ that are constrained by the production of gravitinos and supersymmetric dark matter.
\end{abstract}

DOI: 10.1103/PhysRevD.105.043504

\section{INTRODUCTION}

Successive releases of data on perturbations in the cosmic microwave background (CMB) [1] have provided increasingly strong upper limits on the tensor-to-scalar ratio, $r$, and hence sharpened focus on models of inflation that favor small values of $r$, such as the original Starobinsky model [2] that predicts $r \sim 0.004$ for $55 e$-folds. The recent release of the BICEP/Keck [3] data has followed this trend, imposing the bound $r_{0.05}<0.036$ at the $95 \%$ C.L. where the subscript denotes the pivot scale in $\mathrm{Mpc}^{-1}$. Moreover, the combination of WMAP, Planck, and BICEP/Keck data constrains the scalar tilt to the limited range $0.958<n_{s}<$ 0.975 at the $95 \%$ C.L. for $r=0.004$. A further analysis by [4] used magnetic (BB) autocorrelation data from [5] and allowed a free reionization optical depth and obtained a lower limit on the scalar-to-tensor ratio to $r_{0.05}<0.032$, with a slightly relaxed range on the spectral tilt $0.956<$ $n_{s}<0.974$ at the $95 \%$ C.L. for $r=0.004$.

Published by the American Physical Society under the terms of the Creative Commons Attribution 4.0 International license. Further distribution of this work must maintain attribution to the author(s) and the published article's title, journal citation, and DOI. Funded by SCOAP .
The Starobinsky model is not alone in accommodating the upper limit on $r$. For example, Higgs inflation predicts a similar value of $r$ [6], and similar potentials appear naturally in the context of supergravity, including no-scale supergravity $[7,8]$. In particular, the simplest no-scale supergravity models characterized by a Kähler potential of the form $K=-3 \ln \left(T+\bar{T}-|\phi|^{2} / 3\right)$, where $T$ and $\phi$ are complex scalar fields, predict a Starobinsky-like value of $r$ [9], but the no-scale supergravity framework can also accommodate other possibilities [10].

For example, generalizing $-3 \rightarrow-3 \alpha$ as the coefficient of the logarithm modifies the prediction for $r$ by a factor $\alpha$, as was first pointed out in [11] and subsequently in [12]. Such a modification of the simplest no-scale model is a natural possibility in compactified string models, where $T$ may be interpreted as the volume modulus [13], which is a product of three independent compactification moduli $T_{i}: i=1,2,3$. Models in which inflation is driven by one (two) of these moduli correspond to $\alpha=1 / 3(2 / 3)$ [11]. Larger values of $\alpha$ are also possible, since string compactifications also have complex structure moduli that can contribute to the inflationary dynamics [14].

A common feature of these no-scale supergravity models is a quadratic singularity in the kinetic term for the inflaton. This feature leads generically to an effective potential for the canonically normalized inflaton field with a plateau that 
leads to a quasi-de Sitter inflationary epoch similar to that in Starobinsky inflation. This property was abstracted from the no-scale models in [14], where they were baptized "attractor" models. Two specific types of attractor potential can be distinguished $[11,12,15,16],{ }^{1}$

$$
\begin{aligned}
V= & \frac{3}{4} \lambda M_{P}^{4}\left(1-e^{\left.-\sqrt{\frac{2}{3 \alpha M_{P}}}\right)^{2},}\right. \\
& (\alpha \text {-Starobinsky }[11,12,16]), \\
V= & \frac{3}{4} \lambda M_{P}^{4} \tanh ^{2}\left(\frac{\varphi}{\sqrt{6 \alpha} M_{P}}\right),
\end{aligned}
$$$$
\text { (T Model [15]), }
$$

where $\varphi$ is the canonically normalized inflaton field, $M_{P}=$ $\frac{1}{\sqrt{8 \pi G}} \simeq 2.435 \times 10^{18} \mathrm{GeV}$ the reduced Planck mass, and $\lambda$ the potential scale determined from the CMB normalization and the inflaton field value at horizon crossing. ${ }^{2}$ For the attractor models discussed here, increasing the value of $\alpha$ reduces the flatness of the plateau at the inflaton field value at the horizon crossing of the CMB scale, $\varphi_{*}$, which affects the cosmological observables $n_{s}$ and $r$. It was argued in $[10-12,15,16,18]$ that broad classes of attractor models lead to identical predictions of $n_{s}$ and $r$ in the limit of a large number of $e$-folds, $N_{*}{ }^{3}$. In the context of supergravity, the parameter $\alpha$ determines the curvature of the internal Kähler manifold: $R=2 / \alpha{ }^{4}$

In this paper, we explore the impact of the latest BICEP/ Keck/WMAP/Planck constraints in the $\left(n_{s}, r\right)$ plane on the $\alpha$-Starobinsky and T-model inflationary attractors (see also [20]) from both $[3,4]$. From the analysis in [3], we find that the region of CMB parameters favored at the $68 \%$ C.L. by the combination of CMB data favors $N_{0.05} \gtrsim 50.9(52.6)$ in the $\alpha$-Starobinsky (T models), corresponding to an inflaton decay coupling $y \gtrsim 1.7 \times 10^{-6}\left(1.7 \times 10^{-4}\right)$ for $\alpha=1$, with an order of magnitude sensitivity to $\alpha \in(0.1,5) .^{5}$ In contrast, the analysis in [4] yields substantially weaker

\footnotetext{
${ }^{1}$ We note that $\alpha$-Starobinsky models are also known as $E$ models [17].

${ }^{2}$ The normalization of the potentials is chosen so that the inflaton normalization scale coincides in both cases and is given by Eq. (14). This choice does not affect the CMB observables $n_{s}$ and $r$.

${ }^{3}$ We note that the potentials (1) and (2) are identical at zeroth and first order in $e^{-\sqrt{\frac{2}{3 \alpha M_{P}}}}$ but differ at higher orders and so make different predictions when $\varphi / \sqrt{\alpha}=\mathcal{O}\left(M_{P}\right)$. One could, in principle, consider other attractor potentials that are also equivalent at zeroth and first order, but these are the options commonly considered in the literature.

${ }^{4}$ In general, the Kähler curvature $R$ depends on the total number, $n$, of chiral fields describing the theory $[7,8,16,19]$, $R=n(n+1) / 3 \alpha$, and this result holds for two chiral fields, which is the minimal number needed to construct a plateaulike potential in no-scale supergravity [11].

${ }^{5}$ The corresponding 95\% limits are $N \gtrsim 45.9(47.5)$ and $y \gtrsim 3.8 \times 10^{-13}\left(3.6 \times 10^{-11}\right)$, respectively.
}

bounds, $N_{0.05} \gtrsim 47.9(49.4)$ in the $\alpha$-Starobinsky (T models), corresponding to an inflaton decay coupling $y \gtrsim 1.9 \times$ $10^{-10}\left(1.2 \times 10^{-8}\right)$ for $\alpha=1 .^{6}$ Additionally, supergravity models must avoid overproducing gravitinos and supersymmetric dark matter $[21,22]$. We find that based on [3] $\alpha$ Starobinsky models that respect these constraints fall inside the region favored by the CMB data at the $68 \%$ C.L. only for $\alpha \in(0.67,12)$ and that $\mathrm{T}$ models fall inside this region only for $\alpha \in(1.3,5.1)$. At the $95 \%$ C.L., these ranges are $(0,26)$ and $(0,11)$, respectively. Based on [4], the 68\% C.L. ranges are $(0.4,12)$ and $(0.5,7)$ for the $\alpha$-Starobinsky and $\mathrm{T}$ models, respectively, and the $95 \%$ C.L. ranges are $(0,24)$ and $(0,12)^{7}$

\section{INFLATIONARY DYNAMICS}

The dynamics of the inflaton is characterized by the action

$$
\mathcal{S}=\int d^{4} x \sqrt{-g}\left[\frac{M_{P}^{2}}{2} R+\frac{1}{2} \partial_{\mu} \varphi \partial^{\mu} \varphi-V(\varphi)\right],
$$

where the effective scalar potential is given by Eq. (1) or (2). We use for our analysis the conventional slow-roll parameters, which are given in single-field inflationary models by

$$
\epsilon \equiv \frac{1}{2} M_{P}^{2}\left(\frac{V^{\prime}}{V}\right)^{2}, \quad \eta \equiv M_{P}^{2}\left(\frac{V^{\prime \prime}}{V}\right),
$$

where the prime denotes a derivative with respect to the inflaton field, $\varphi$. In the slow-roll approximation, the number of $e$-folds can be computed using

$$
N_{*} \simeq \frac{1}{M_{P}^{2}} \int_{\varphi_{\text {end }}}^{\varphi_{*}} \frac{V(\varphi)}{V^{\prime}(\varphi)} d \varphi \simeq \int_{\varphi_{\text {end }}}^{\varphi_{*}} \frac{1}{\sqrt{2 \epsilon}} \frac{d \varphi}{M_{P}},
$$

where $k_{*}=0.05 \mathrm{Mpc}^{-1}$ is the pivot scale used in the Planck analysis. The end of inflation occurs when $\ddot{a}=0$, i.e., $\dot{\varphi}_{\text {end }}^{2}=V\left(\varphi_{\text {end }}\right)$.

The principal CMB observables, namely, the scalar tilt, $n_{s}$, the tensor-to-scalar ratio, $r$, and the amplitude of the curvature power spectrum, $A_{S}$, can be expressed as follows in terms of the slow-roll parameters:

$$
\begin{aligned}
n_{s} & \simeq 1-6 \epsilon_{*}+2 \eta_{*}, \\
r & \simeq 16 \epsilon_{*}, \\
A_{S *} & \simeq \frac{V_{*}}{24 \pi^{2} \epsilon_{*} M_{P}^{4}},
\end{aligned}
$$

\footnotetext{
${ }^{6}$ In this case, the corresponding $95 \%$ C.L. are $N \gtrsim 42.9(44.6)$ and $y \gtrsim 2.8 \times 10^{-17}\left(4.0 \times 10^{-15}\right)$, respectively.

${ }^{7}$ Here, the lower bound $\alpha>0$ arises because $\alpha=0$ leads to a completely flat potential that is not suitable for inflation.
} 
where $V_{*}=V\left(\varphi_{*}\right)$ and $A_{S *} \simeq 2.1 \times 10^{-9}$ [1]. In the large $N_{*}$ limit, the inflationary attractor potentials (1) and (2) predict [11]

$$
n_{s} \simeq 1-\frac{2}{N_{*}}, \quad r \simeq \frac{12 \alpha}{N_{*}^{2}},
$$

where the approximation holds for $\alpha \lesssim \mathcal{O}(1)$ in $\alpha$ Starobinsky models, and the full analytical expression can be found in [22].

Using expression (5), we can calculate the approximate value of the inflaton field at the horizon exit scale $k_{*}$ [23] when $\alpha=1$,

$$
\begin{gathered}
\frac{\varphi_{*}}{M_{P}} \simeq \sqrt{\frac{3}{2}}\left[1+\frac{3}{4 N_{*}-3}\right] \ln \left(\frac{4 N_{*}}{3}+e^{\sqrt{\frac{2}{3} \varphi_{\text {end }}}}-\sqrt{\frac{2}{3} \frac{\varphi_{\text {end }}}{M_{P}}}\right), \\
(\alpha \text {-Starobinsky }),
\end{gathered}
$$

$$
\left.\frac{\varphi_{*}}{M_{P}} \simeq \sqrt{\frac{3}{2}} \cosh ^{-1}\left(\frac{4 N_{*}}{3}+\cosh \left(\sqrt{\frac{2}{3}} \frac{\varphi_{\text {end }}}{M_{P}}\right)\right), \quad \text { (T Model }\right),
$$

with

$$
\begin{array}{ll}
\frac{\varphi_{\text {end }}}{M_{P}} \simeq \sqrt{\frac{3}{2}} \ln \left[\frac{2}{11}(4+3 \sqrt{3})\right], & (\alpha \text {-Starobinsky }), \\
\frac{\varphi_{\text {end }}}{M_{P}} \simeq \sqrt{\frac{3}{2}} \ln \left[\frac{1}{11}(14+5 \sqrt{3})\right], & (\text { T Model }),
\end{array}
$$

where $\varphi_{\text {end }}$ was calculated using the expression $\epsilon=(1+\sqrt{1-\eta / 2})^{2}$, and the full analytical approximations for $\varphi_{*}$ and $\varphi_{\text {end }}$ can be found in the Appendix A, where they are given by Eqs. (A2)-(A5). Combining the expressions above with expression (8) for the curvature power spectrum, we find that the inflaton normalization scale is proportional to $\lambda$, which is in turn proportional to $\alpha$ and given by

$$
\lambda \simeq \frac{24 \alpha \pi^{2} A_{S *}}{N_{*}^{2}} .
$$

We now calculate the number of $e$-folds, $N_{*}$, assuming that there is no additional entropy injection between the end of reheating and when the horizon scale $k_{*}$ reenters the horizon [24,25],

$$
\begin{aligned}
N_{*}= & \ln \left[\frac{1}{\sqrt{3}}\left(\frac{\pi^{2}}{30}\right)^{1 / 4}\left(\frac{43}{11}\right)^{1 / 3} \frac{T_{0}}{H_{0}}\right]-\ln \left(\frac{k_{*}}{a_{0} H_{0}}\right)-\frac{1}{12} \ln g_{\text {reh }} \\
& +\frac{1}{4} \ln \left(\frac{V_{*}^{2}}{M_{P}^{4} \rho_{\text {end }}}\right)+\frac{1-3 w_{\text {int }}}{12\left(1+w_{\text {int }}\right)} \ln \left(\frac{\rho_{\text {rad }}}{\rho_{\text {end }}}\right)
\end{aligned}
$$

where the present Hubble parameter and photon temperature are given by $H_{0}=67.36 \mathrm{~km} \mathrm{~s}^{-1} \mathrm{Mpc}^{-1}$ [26] and
$T_{0}=2.7255 \mathrm{~K}$ [27]. Here, $\rho_{\text {end }}$ and $\rho_{\text {rad }}$ are the energy density at the end of inflation and at the beginning of the radiation domination era when $w=p / \rho=1 / 3$, respectively, and $a_{0}=1$ is the present day scale factor and $g_{\text {reh }}=$ $915 / 4$ the effective number of relativistic degrees of freedom in the minimal supersymmetric standard model at the time of reheating. The equation of state parameter averaged over the $e$-folds during reheating is

$$
w_{\text {int }} \equiv \frac{1}{N_{\text {rad }}-N_{\text {end }}} \int_{N_{\text {end }}}^{N_{\text {rad }}} w(n) d n .
$$

Using the numerical values given above with the Planck pivot scale $k_{*}=0.05 \mathrm{Mpc}^{-1}$, we find the following value for the sum of the first two lines in (15): $N_{*} \simeq 61.04+\cdots$. Mechanisms for producing a baryon asymmetry (such as leptogenesis) are simplified when $T_{\text {reh }} \gtrsim$ the electroweak scale. Accordingly, we also display results for a reheating temperature $T_{\text {reh }}=T_{\mathrm{EW}} \sim 100 \mathrm{GeV}$, while acknowledging that lower reheating temperatures are possible. For $T_{\text {reh }}=T_{\mathrm{EW}}$, we take the Standard Model value for $g_{\mathrm{reh}}=427 / 4$ and find $N_{\mathrm{EW}}=61.10+\cdots$. The minimum reheating temperature that is compatible with big bang nucleosynthesis $(\mathrm{BBN})$ is $T_{\text {reh }} \gtrsim \mathcal{O}(1) \mathrm{MeV}$. Using $T_{\mathrm{BBN}}=2 \mathrm{MeV}$ in our numerical analysis, corresponding to $g_{\text {reh }}=10.75$, the sum of the first two lines of (15) takes the following numerical value: $N_{\mathrm{BBN}} \simeq 61.29+\cdots$.

To calculate the values of $N_{*}, N_{\mathrm{EW}}$, and $N_{\mathrm{BBN}}$ numerically, we use the following equations that govern the cosmic background dynamics:

$$
\begin{aligned}
\dot{\rho}_{\varphi}+3 H \rho_{\varphi} & =-\Gamma_{\varphi} \rho_{\varphi}, \\
\dot{\rho}_{r}+4 H \rho_{r} & =\Gamma_{\varphi} \rho_{\varphi}, \\
\rho_{\varphi}+\rho_{r} & =3 M_{P}^{2} H^{2}, \\
\frac{d}{d t}\left(N w_{\text {int }}\right) & =H w,
\end{aligned}
$$

where $\rho_{\varphi}$ and $\rho_{r}$ are the energy densities of the inflaton and produced radiation, respectively, and $\Gamma_{\varphi}$ is the inflaton decay rate given by

$$
\Gamma_{\varphi}=\frac{y^{2}}{8 \pi} m_{\varphi},
$$

where $y$ is a Yukawa-like coupling, and we find the following masses in the inflationary attractor potentials (1) and (2),

$$
m_{\varphi}=\sqrt{\frac{\lambda}{\alpha}} M_{P}, \quad(\alpha \text {-Starobinsky }),
$$

\footnotetext{
${ }^{8}$ We note that when we calculate the tensor-to-scalar ratio $r_{0.002}$ numerically, we evaluate $N_{*}$ at the pivot scale $k_{*}=0.002 \mathrm{Mpc}^{-1}$.
} 


$$
m_{\varphi}=\frac{1}{2} \sqrt{\frac{\lambda}{\alpha}} M_{P}
$$

(T Model).

\section{REHEATING}

The reheating process occurs after the end of inflation in a matter-dominated background. As the inflaton starts to decay, the dilute plasma reaches a maximum temperature, $T_{\max }$ [28,29], and subsequently starts falling as $T \propto a^{-3 / 8}$. The reheating temperature is defined through $[30,31]$

$$
\frac{\pi^{2} g_{\mathrm{reh}} T_{\mathrm{reh}}^{4}}{30}=\frac{12}{25}\left(\Gamma_{\varphi} M_{P}\right)^{2}
$$

when the energy density of the inflaton is equal to the energy density of radiation, corresponding to

$T_{\text {reh }} \simeq 1.9 \times 10^{15} \mathrm{GeV} \cdot y \cdot g_{\text {reh }}^{-1 / 4}\left(\frac{m_{\varphi}}{3 \times 10^{13} \mathrm{GeV}}\right)^{1 / 2}$.

In order to evaluate the constraint on $T_{\text {reh }}$ from overproduction of supersymmetric dark matter in scenarios where the gravitino is lighter than $T_{\text {reh, }}$ we use the expression $[29,32]^{9}$

$$
Y_{3 / 2}(T)=0.00336\left(1+0.51 \frac{m_{1 / 2}^{2}}{m_{3 / 2}^{2}}\right)\left(\frac{\Gamma_{\varphi}}{M_{p}}\right)^{1 / 2},
$$

where $Y_{3 / 2} \equiv n_{3 / 2} / n_{\text {rad }}$ is the gravitino yield, $n_{\text {rad }}=\zeta(3) T^{3} / \pi^{2}, m_{3 / 2}$ the gravitino mass, and $m_{1 / 2}$ the gluino mass [33-35]. Disregarding the term $m_{1 / 2}^{2} / m_{3 / 2}^{2}$ in (26) and using the observed dark matter density today, $\Omega_{\mathrm{CDM}} h^{2} \simeq 0.12$, we find the following upper limit on the Yukawa-like inflaton coupling, assuming that the gravitino decays after the lightest supersymmetric particle (LSP) decouples,

$$
|y|<9.2 \times 10^{-8} \sqrt{\frac{M_{P}}{m_{\varphi}}}\left(\frac{100 \mathrm{GeV}}{m_{\mathrm{LSP}}}\right),
$$

where $m_{\mathrm{LSP}}$ is the mass of the LSP, and the inflaton masses for the different inflationary attractor potentials are given by Eqs. (22) and (23). ${ }^{10}$ We note that since $m_{\varphi} \propto 1 / \sqrt{\alpha},|y| \propto \alpha^{1 / 4}{ }^{11}$

\footnotetext{
${ }^{9} \mathrm{We}$ use here an analytical approximation since there is only a $0.03 \%$ difference between the analytical and fully numerical calculation.

${ }^{10}$ If the gravitino is the LSP, the second term in the brackets in (26) must be taken into account, and the constraint on $y$ depends on the ratio $m_{1 / 2} / m_{3 / 2}$.

${ }^{11}$ For another recent analysis of gravitino constraints in light of the BICEP/Keck results, see [36].
}

In high-scale supersymmetry models in which the gravitino mass may be significantly larger than the electroweak scale and the other supersymmetric particles are heavier than the inflaton, the gravitino, which is now the LSP, is pair produced via its longitudinal components [37]. In such a scenario, we find [38]

$$
\begin{aligned}
\Omega_{3 / 2} h^{2} \simeq & 0.12\left(\frac{|y|}{3.0 \times 10^{-7}}\right)^{19 / 5}\left(\frac{m_{\varphi}}{3 \times 10^{13} \mathrm{GeV}}\right)^{67 / 10} \\
& \times\left(\frac{0.1 \mathrm{EeV}}{m_{3 / 2}}\right)^{3}\left(\frac{0.030}{\alpha_{3}}\right)^{16 / 5}
\end{aligned}
$$

where $m_{3 / 2}$ is the gravitino mass, and $\alpha_{3}$ is the strong coupling. Using the observed dark matter abundance today to constrain $\Omega_{3 / 2} h^{2}$, we find that avoiding overproduction of dark matter imposes the following bound:

$$
|y|<6.6 \times 10^{-16}\left(\frac{M_{P}}{m_{\varphi}}\right)^{67 / 38}\left(\frac{m_{3 / 2}}{0.1 \mathrm{EeV}}\right)^{15 / 19} .
$$

We note that in a nonsupersymmetric theory there would, in general, be a lower limit on $y$ due to the fact that it generates radiative corrections $\propto y^{4}$ in the effective inflaton potential [39]. However, this is not the case in supersymmetric models such as those discussed above, where these radiative corrections cancel down to the level of the relatively small supersymmetry-breaking effects [40].

\section{RESULTS}

We solve the cosmic background equations (17)-(20) numerically to determine the number of $e$-folds $N_{*}, N_{\mathrm{EW}}$, and $N_{\mathrm{BBN}}$. In the $\alpha=1$ case, the procedure of calculating the analytical approximations for $N_{*}$ is given in Appendix A [see Eqs. (A11) and (A12)]. The full numerical computation of the $\mathrm{CMB}$ observables is discussed in Appendix B.

Figure 1 summarizes our numerical results based on the analysis of [3]: those for $\alpha$-Starobinsky models are shown in the upper pair of panels and those for T models in the lower pair. For each of the two models, we derive limits on $N_{*}$ from the requirements that $T_{\text {reh }}>2 \mathrm{MeV}(100 \mathrm{GeV})$ and the supersymmetric relic density when $m_{\mathrm{LSP}}=$ $100 \mathrm{GeV}$. The former gives a lower limit to $N_{*}$, while the latter gives an upper limit. We also derive the corresponding limits on $y$. These are compared to the $68 \%$ and 95\% C.L. on $N$ and $y$ from the BICEP/Keck constraints on $n_{s}$. For $\alpha=1$, we find the following limits: 

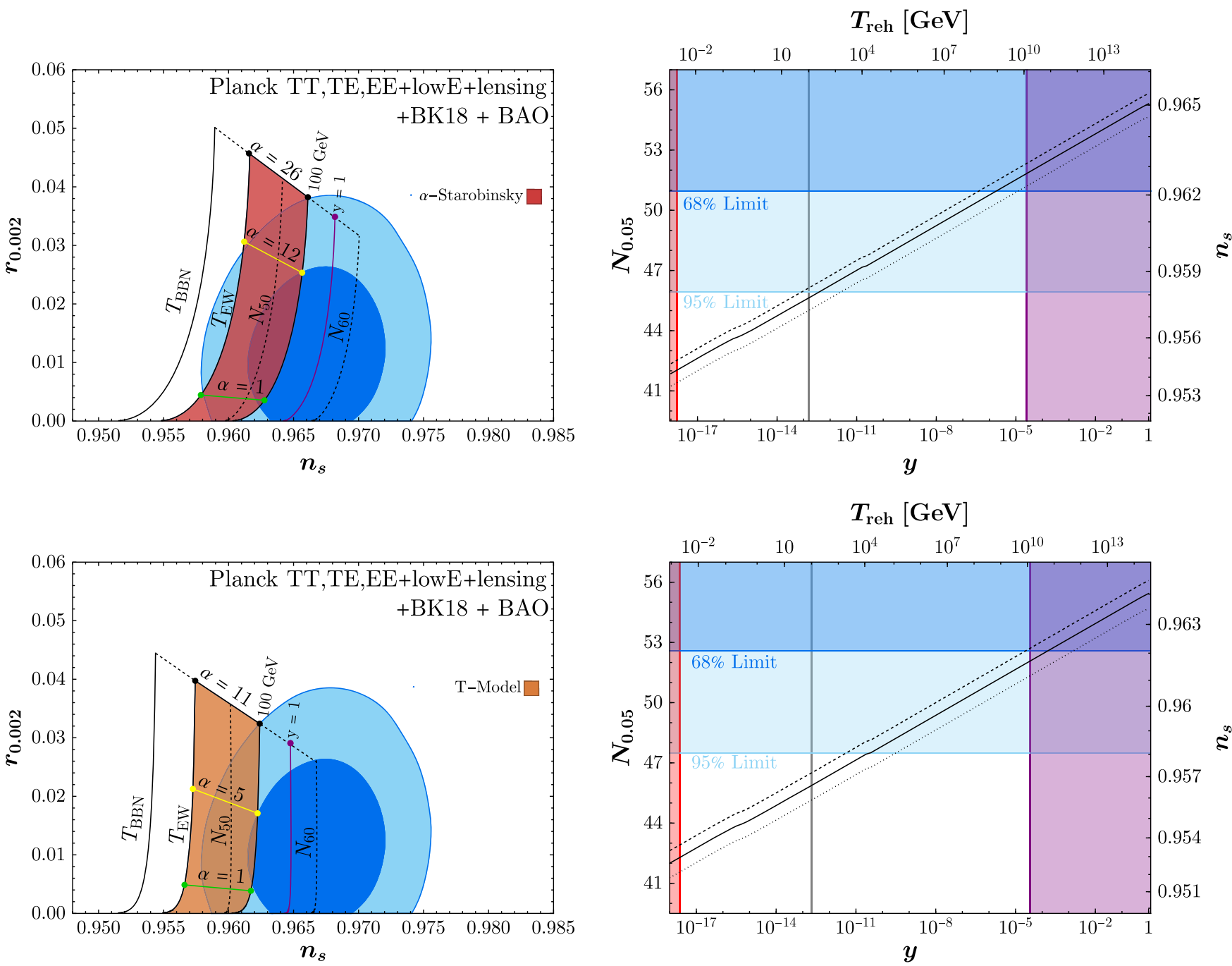

FIG. 1. Illustrations of the impacts of the BICEP/Keck and other constraints on $\alpha$-Starobinsky models (upper panels) and T models (lower panels) based on the analysis of [3]. The left panels compare the observational 68\% and 95\% C.L. constraints in the $\left(n_{s}, r\right)$ plane (using pivot scales 0.002 for $r$ and 0.05 for $n_{s}$ ) with the model predictions for different numbers of $e$-folds $N_{50,60}$, showing also the predictions for an inflaton coupling $y=1$, the constraints from $T_{\text {reh }} \geq T_{\mathrm{BBN}}$ and $T_{\mathrm{EW}}$, and the constraints if the LSP mass is $100 \mathrm{GeV}$. The right panels display $(y, N)$ planes (using the pivot scale 0.05), showing the relations between $y$ and $T_{\text {reh }}$ and between $N$ and $n_{s}$, and the values $\alpha=0.1,1,5$ (dashed, solid, and dotted black lines). We also include lower limits on $y$ from BBN (red line), $T_{\text {reh }}=T_{\mathrm{EW}}$ (gray line), and gravitino production (purple line) for $\alpha=1$, which increase for smaller $\alpha$, and $68 \%$ and $95 \%$ C.L. lower limits on $N_{0.05}$ from $\mathrm{BICEP} /$ Keck and other data (blue lines).

$\alpha$-Starobinsky:

$41.8(45.6)<N_{*}<51.8$,

$1.7 \times 10^{-18}\left(1.6 \times 10^{-13}\right)<|y|<2.6 \times 10^{-5}$,

$N_{68 \%}=50.9, \quad N_{95 \%}=45.9$,

$T_{\text {reh, } 68 \%}=8.7 \times 10^{8} \mathrm{GeV}, \quad T_{\text {reh, }, 95 \%}=2.4 \times 10^{2} \mathrm{GeV}$,

$y_{68 \%}=1.7 \times 10^{-6}, \quad y_{95 \%}=3.8 \times 10^{-13}$,
T Model:

$42.0(45.8)<N_{*}<52.1$,

$2.3 \times 10^{-18}\left(2.2 \times 10^{-13}\right)<|y|<3.6 \times 10^{-5}$,

$N_{68 \%}=52.6, \quad N_{95 \%}=47.5$,

$T_{\text {reh, } 68 \%}=5.9 \times 10^{10} \mathrm{GeV}, \quad T_{\text {reh, } 95 \%}=1.4 \times 10^{4} \mathrm{GeV}$,

$y_{68 \%}=1.7 \times 10^{-4}, \quad y_{95 \%}=3.6 \times 10^{-11}$. 
We note that the first two lines do not depend on the BICEP/ Keck constraints, since these limits are derived from the conditions $T_{\text {reh }}>2 \mathrm{MeV}(100 \mathrm{GeV})$ (smaller limit) and $m_{\mathrm{LSP}}=100 \mathrm{GeV}$ (larger limit). The dark (light) blue regions in the left panels are the 68\% (95)\% C.L. regions of the $\left(n_{s}, r_{0.002}\right)$ planes favored by a global analysis of the $\mathrm{CMB}$ and baryon acoustic oscillations (BAO) data. We also show in the left panels of Fig. 1 dotted contours corresponding to 60 and $50 e$-folds, solid lines corresponding to the maximum number of $e$-folds consistent with $y \leq 1$, and the minimum number of $e$-folds consistent with $T_{\text {reh }}>T_{\mathrm{BBN}}$ and $T_{\mathrm{EW}}$, as well as the dark matter density constraints for a LSP mass of $100 \mathrm{GeV}$. The corresponding limit for a gravitino mass of $10^{8} \mathrm{GeV}$ in the high-scale supersymmetry case would lie roughly midway between the $m_{\text {LSP }}=$ $100 \mathrm{GeV}$ and $N_{*}=50$ lines. For the $\alpha$-Starobinsky ( $\mathrm{T}$ models) we shade in red (orange) the preferred region respecting the constraint $T_{\text {reh }}>T_{\mathrm{EW}}$ and the relic density constraint with $m_{\mathrm{LSP}}=100 \mathrm{GeV}$. In the upper left panel, we also show lines corresponding to $\alpha=1$ and 12 , the latter being the largest value allowed at the $68 \%$ C.L. for $m_{\mathrm{LSP}}=100 \mathrm{GeV}$, and $\alpha=26$, the largest value allowed at the $95 \%$ C.L. for $m_{\mathrm{LSP}}=100 \mathrm{GeV}$. We see in the lower left panel that values of $\alpha \in(1.3,5.1)$ are consistent with the data at the $68 \%$ C.L. if $m_{\mathrm{LSP}}=100 \mathrm{GeV}$, and values of $\alpha \leq 11$ are allowed at the $95 \%$ C.L.

The right panels of Fig. 1 show the $\left[y\left(T_{\text {reh }}\right), N_{0.05}\left(n_{s}\right)\right]$ planes for the $\alpha$-Starobinsky models and T models. The left-most vertical lines (red) correspond to the minimum values of $y$ allowed by BBN, the middle vertical lines (gray) to $T_{\text {reh }}=T_{\mathrm{EW}}$, and the right-most vertical lines (purple) to the maximum values allowed for $m_{\mathrm{LSP}}=100 \mathrm{GeV}$. We assume $\alpha=1$ when plotting the parameters and constraints. The constraints would each move to the right (towards larger values of $y$ and $T_{\text {reh }}$ ) with decreasing values of $\alpha$, although their dependencies are weak. The diagonal lines are the predictions of the $\alpha$-Starobinsky and T models for $\alpha=0.1$ (dashed lines), 1 (solid lines), and 5 (dotted lines). Finally, we show as horizontal lines the lower limits on $n_{0.05}$ at the $68 \%$ and $95 \%$ C.L. We see that the $68 \%$ lower limit of $N_{0.05}$ requires $y>1.7 \times 10^{-6}$ in the $\alpha$-Starobinsky model and $y>1.7 \times$ $10^{-4}$ for the T-Starobinsky model, both for $\alpha=1$. This implies a lower limit to the reheating temperature of $8.7 \times$ $10^{8}$ and $5.9 \times 10^{10} \mathrm{GeV}$ for the $\alpha$-Starobinsky models and $\mathrm{T}$ models, respectively. This limit is relaxed at the 95\% C.L., where the lower limit on the reheating temperature drops to $2.4 \times 10^{2} \mathrm{GeV}$ in the $\alpha$-Starobinsky models and $1.4 \times 10^{4} \mathrm{GeV}$ for the $\mathrm{T}$ models.

We assumed in the above analysis that generation of a factor $\Delta$ of entropy subsequent to inflaton decay could be neglected. However, this may not be the case, e.g., in models with additional phase transitions at temperatures between $T_{\text {reh }}$ and $T_{\mathrm{EW}}$, such as those based on flipped SU(5) grand unified theories (GUTs) [41]. In this case, there would be a modification to the calculation of $N_{*}$ in Eq. (15) in the form of an extra term $-\frac{1}{3} \ln \Delta$ in the righthand side. This would in turn modify the left panels of Fig. 1; e.g., the $T_{\mathrm{BBN}}$ and $T_{\mathrm{EW}}$ constraints would move to lower $n_{s}$, as would the $y=1$ line, whereas the $N_{50}$ and $N_{60}$ lines would be unchanged, as would the LSP density constraint. As entropy generation would allow a higher initial gravitino abundance, and thus a higher reheating temperature, the contribution to $N_{*}$ from reheating is exactly compensated by the contribution from $\Delta$. In addition, the lines of fixed $\alpha$ are unchanged. The net result would be to expand the favored regions of the $\left(n_{s}, r_{0.002}\right)$ planes towards lower values of $n_{s}$, while keeping the same overlaps with the regions of the planes favored by the BICEP/Keck and other constraints at the $68 \%$ C.L. However, this would require higher reheating temperatures.

Figure 2 shows analogous results based on the analysis in [4]. Since this work provides limits on $r$ using $0.05 \mathrm{Mpc}^{-1}$ for the pivot scale, we have recalculated the theory curves accordingly, although the difference is quite small. What is more striking is the difference in the $68 \%$ and $95 \%$ lower limits to $n_{s}$. These are shifted slightly to smaller values, and, as one can see in Fig. 2, a large portion of the red-shaded region (between $T_{\mathrm{EW}}$ and the $100 \mathrm{GeV}$ relic density limit) now overlaps the $68 \%$ C.L. observational region (dark blue). In the right panels, we see that the weaker lower limits on $n_{s}$ reduce the lower limits on $N_{0.05}$ and hence allow a smaller inflaton coupling to matter and a lower reheat temperature. However, the allowed ranges for $\alpha$ are only slightly modified: $(0.4,12)$ and $(0,24)$ for the $\alpha$ Starobinsky model at $68 \%$ and $95 \%$ C.L., respectively, and $(0.5,7)$ and $(0,12)$ for the T model.

The modified limits analogous to Eqs. (30) and (31) for $\alpha=1$ are

$\alpha$-Starobinsky:

$N_{68 \%}=47.9, \quad N_{95 \%}=42.9$,

$T_{\text {reh, } 68 \%}=9.8 \times 10^{4} \mathrm{GeV}, \quad T_{\text {reh, } 95 \%}=0.031 \mathrm{GeV}$,

$y_{68 \%}=1.9 \times 10^{-10}, \quad y_{95 \%}=2.8 \times 10^{-17}$,

T Model:

$N_{68 \%}=49.4, \quad N_{95 \%}=44.6$,

$T_{\text {reh, } 68 \%}=4.4 \times 10^{6} \mathrm{GeV}, \quad T_{\text {reh, } 95 \%}=2.0 \mathrm{GeV}$,

$y_{68 \%}=1.2 \times 10^{-8}, \quad y_{95 \%}=4.0 \times 10^{-15}$.

The limits on $N_{*}$ and $y$ from limits to $T_{\text {reh }}$ and the relic density are unaffected by the choice of data analysis and are not repeated.

\section{DISCUSSION}

As can be seen from the left panels of Figs. 1 and 2, the primary driver of the upper limits on $\alpha$ is the new upper limit on $r$, whereas the constraint on $n_{s}$ is the primary driver 

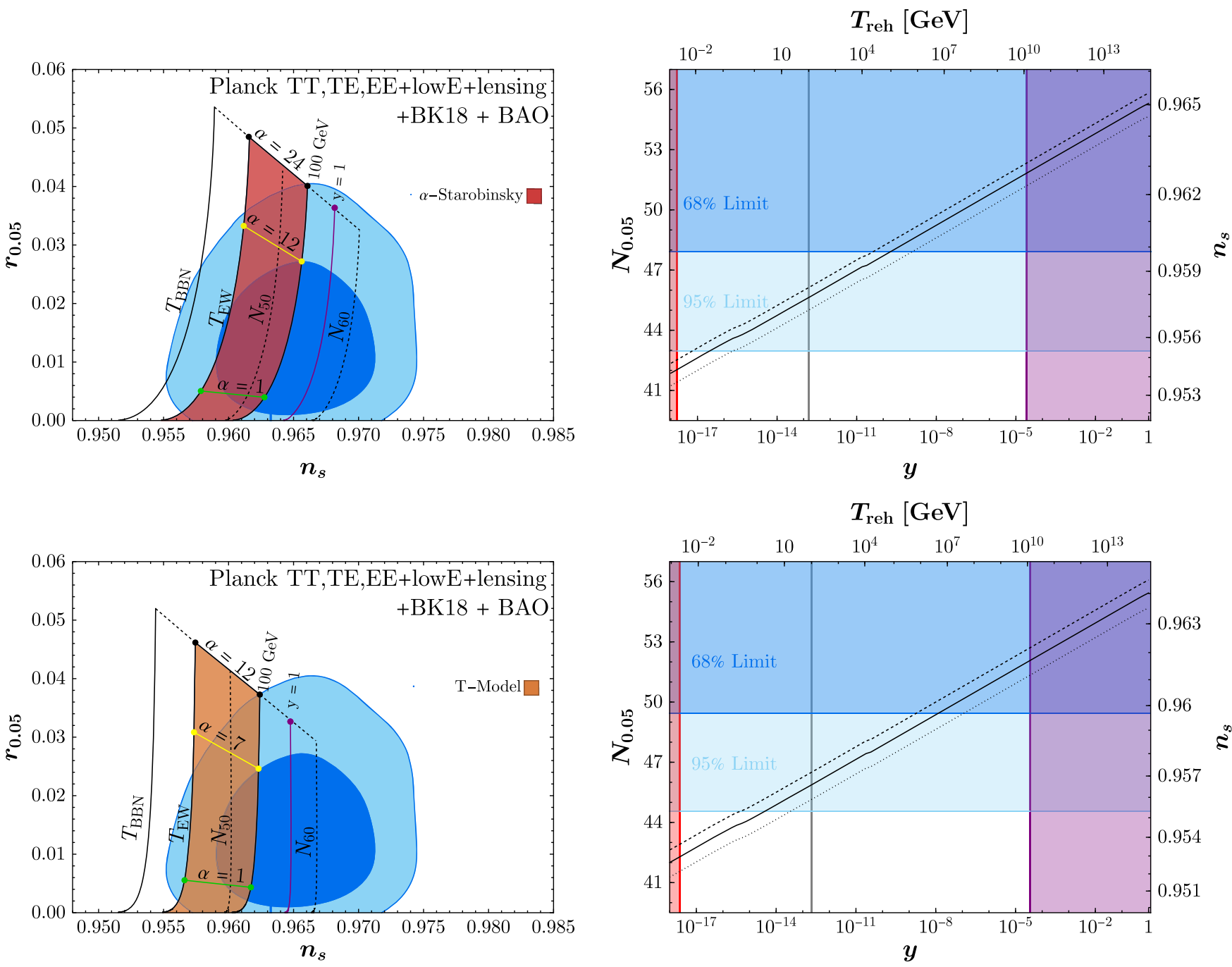

FIG. 2. Illustrations of the impacts of the BICEP/Keck and other constraints on $\alpha$-Starobinsky models (upper panels) and T models (lower panels) based on the analysis of [4]. The left panels compare the observational 68\% and 95\% C.L. constraints in the $\left(n_{s}, r\right)$ plane (using pivot scales 0.05 for both $r$ and $n_{s}$ ) with the model predictions for different numbers of $e$-folds $N_{50,60}$, showing also the predictions for an inflaton coupling $y=1$, the constraints from $T_{\text {reh }} \geq T_{\mathrm{BBN}}$ and $T_{\mathrm{EW}}$, and the constraints if the LSP mass is $100 \mathrm{GeV}$. The right panels display $(y, N)$ planes (using the pivot scale 0.05), showing the relations between $y$ and $T_{\text {reh }}$ and between $N$ and $n_{s}$, and the values $\alpha=0.1,1,5$ (dashed, solid, and dotted black lines). We also include lower limits on $y$ from BBN (red line), $T_{\text {reh }}=T_{\mathrm{EW}}$ (gray line), and gravitino production (purple line) for $\alpha=1$, which increase for smaller $\alpha$, and $68 \%$ and 95\% C.L. lower limits on $N_{0.05}$ from $\mathrm{BICEP} /$ Keck and other data (blue lines).

of the lower limit on the number of $e$-folds. In both the $\alpha$ Starobinsky and T models there is also an upper limit on the number of $e$-folds due to requiring the inflaton decay coupling $y \lesssim \mathcal{O}(1)$, namely, $N_{*} \lesssim 56$, as seen in the right panels of the figures, which restricts $n_{s}$ to the left halves of the preferred ovals in the left panels of Figs. 1 and 2. In both cases, couplings near or at this upper limit lead to observables closest to the central value of the confidence contours. This indicates that the updated constraints in $n_{s}$ favor scenarios for which radiation domination is almost immediately reached after the end of inflation. We note that such a thermal history is always realized regardless of the inflaton-Standard Model couplings if the inflationary potential is quartic near its minimum, as is the case of Higgs inflation [6], WIMPflation [42], or T models of the form $V \sim \tanh ^{4}\left(\varphi / \sqrt{6 \alpha} M_{P}\right)$ [30,43]. For quartic minima, $N_{*} \simeq 56$, independent of the reheating temperature.

The values of the effective Yukawa coupling $y$ disfavored by electroweak scale gravitino overproduction, shown in purple in Figs. 1 and 2, correspond coincidentally to the domain of nonperturbative particle production (preheating). Indeed, for $y \gtrsim 10^{-5}$, efficient parametric resonance will be present during the early stages of reheating, for either fermionic or bosonic inflaton decay products [44-48]. However, this effect is not necessarily reflected in the CMB observables. In the case of fermionic preheating, the 
expansion history during reheating (and hence, $w_{\text {int }}$ and $\left.\rho_{\text {rad }}\right)$ is not affected unless $y \sim \mathcal{O}(1)$. The resulting Pauli suppression of particle production simply reduces the energy density of radiation relative to the value predicted by (18) for a time much shorter than the duration of reheating [48]. Hence, our results for $N_{*}$ shown in the left panels of Fig. 1 would be mostly unchanged in this fermionic case. In the case of bosonic preheating, the efficiency of nonperturbative particle production depends on the resonance band structure of the coupling. If the backreaction regime is reached, transient radiation-dominated stages can occur during reheating, modifying $w_{\text {int }}$ and hence our predictions $[48,49]$. However, we do not delve here into this model-dependent issue. Finally, for attractors with quadratic minima, the self-interaction of the inflaton does not disrupt the matterlike oscillation of the inflaton condensate during reheating [50].

Turning to the future, we note that the experiments CMB-S4 [51] and LiteBIRD [52] will target primarily the search for $\mathrm{B}$ modes in the $\mathrm{CMB}$ and will impose strong constraints on $r$, with the potential to reduce substantially the uncertainty in $r$, by a factor $\mathcal{O}(2)$. Such a measurement will reduce the uncertainty in $\alpha$ to a similar value, constraining significantly string models of inflation. Unfortunately, the ability of these experiments to constrain $n_{s}$ is limited. However, this is an important objective for the future, as $n_{s}$ is related directly to the magnitude of the coupling between the inflaton and matter, whose understanding will be key for connecting the theory of inflation to laboratory physics.

\section{ACKNOWLEDGMENTS}

We thank Marco Drewes, Mathias Pierre, Douglas Scott, and Matthieu Tristram for helpful discussions. The work of J. E. was supported partly by the United Kingdom STFC Grant No. ST/T000759/1 and partly by the Estonian Research Council via a Mobilitas Pluss grant. J.E., M. A. G. G., and S. V. acknowledge the hospitality of the Institut Pascal at the Universite Paris-Saclay during the 2021 Paris-Saclay Astroparticle Symposium, with the support of the P2IO Laboratory of Excellence program "Investissements d'avenir" ANR-11-IDEX-0003-01 ParisSaclay and ANR-10-LABX-0038, and the P2I axis of the Graduate School Physics of the Université Paris-Saclay, as well as Laboratoire de Physique des 2 infinis Irène JoliotCurie (IJCLab), Commissariat à l'Energie Atomique (CEA), Institut de Physique Théorique (IPhT), Astroparticle Physics European Consortium (APPEC), and European Consortium for Astroparticle Theory (EuCAPT) ANR-11-IDEX-0003-01 Paris-Saclay and ANR-10-LABX-0038. M. A. G. G. was also supported by the IN2P3 master project UCMN. The work of D. V. N. was supported partly by DOE Grant No. DE-FG02-
13ER42020 and partly by the Alexander S. Onassis Public Benefit Foundation. The work of K. A. O. was supported in part by DOE Grant No. DE-SC0011842 at the University of Minnesota.

\section{APPENDIX A: ANALYTICAL APPROXIMATIONS}

As stated in the main text, the power spectrum and reheating constraints summarized in Fig. 1 have been obtained numerically. In this Appendix we provide analytical approximations to the relevant inflationary quantities.

The end of inflation corresponds to the end of the epoch of accelerated expansion, i.e., $\ddot{a}=0$ or $\epsilon_{H}=1$, where $\epsilon_{H}=-\dot{H} / H^{2}$ is the first Hubble flow function. In terms of the potential slow-roll parameters (4), it can be shown that the end of inflation occurs approximately when [23]

$$
\epsilon \simeq(1+\sqrt{1-\eta / 2})^{2} .
$$

This expression can be used to obtain the following closedform estimates for the value of the inflaton field at the end of inflation for $\alpha$-Starobinsky models,

$$
\frac{\varphi_{\text {end }}}{M_{P}} \simeq \sqrt{\frac{3 \alpha}{2}} \ln \left[\frac{2(6 \alpha+3 \sqrt{3 \alpha}-2)}{12 \alpha-1}\right],
$$

and for T models,

$$
\begin{aligned}
\frac{\varphi_{\text {end }}}{M_{P}} \simeq & \sqrt{\frac{3 \alpha}{2}} \ln \left[\frac{4-6 \sqrt{\alpha(5+4 \alpha)}}{1-12 \alpha}\right. \\
& +\sqrt{\left.\frac{75}{5+68 \alpha+16 \sqrt{\alpha(5+4 \alpha)}}\right]} .
\end{aligned}
$$

As expected, for $\alpha=1$, we recover Eqs. (12) and (13). Compared to the exact values, the analytic approximations have errors of $2 \%(2 \%, 4 \%)$ for $\alpha=1(0.1,10)$ in the case of $\alpha$-Starobinsky models and of 5\% (3\%, 5\%) for $\alpha=1(0.1$, 10) for $T$ models.

The value of the inflaton field at the moment when the pivot scale crosses the horizon can be estimated by integrating Eq. (5). In the case of $\alpha$-Starobinsky models,

$$
\begin{aligned}
\frac{\varphi_{*}}{M_{P}} \simeq & \sqrt{\frac{3 \alpha}{2}}\left[1+\frac{3 \alpha}{4 N_{*}-3 \alpha}\right] \\
& \times \ln \left(\frac{4 N_{*}}{3 \alpha}+e^{\sqrt{\frac{\sqrt{2} \varphi_{\text {end }}}{3 M_{P}}}}-\sqrt{\frac{2}{3}} \frac{\varphi_{\text {end }}}{M_{P}}\right),
\end{aligned}
$$

and for T models,

$$
\frac{\varphi_{*}}{M_{P}} \simeq \sqrt{\frac{3 \alpha}{2}} \cosh ^{-1}\left[\frac{4 N_{*}}{3 \alpha}+\cosh \left(\sqrt{\frac{2}{3 \alpha}} \frac{\varphi_{\text {end }}}{M_{P}}\right)\right] .
$$


For $40<N_{*}<60$, the relative errors are at most $0.3 \%$ $(0.3 \%, 3 \%)$ for $\alpha=1(0.1,10)$ in the $\alpha$-Starobinsky case and $0.5 \%(0.4 \%, 0.7 \%)$ for $\alpha=1(0.1,10)$ in the case of T-model inflation.

The logarithm of the so-called reheating parameter [25],

$$
\begin{gathered}
\ln R_{\mathrm{rad}} \equiv \ln \left[\frac{a_{\mathrm{end}}}{a_{\mathrm{rad}}}\left(\frac{\rho_{\mathrm{end}}}{\rho_{\mathrm{rad}}}\right)^{1 / 4}\right] \\
=\frac{1-3 w_{\mathrm{int}}}{12\left(1+w_{\mathrm{int}}\right)} \ln \left(\frac{\rho_{\mathrm{rad}}}{\rho_{\mathrm{end}}}\right)
\end{gathered}
$$

may be estimated by noting that the energy density of the relativistic inflaton decay products, assuming a constant decay rate $\Gamma_{\varphi}$, can be written as [23]

$$
\rho_{\mathrm{rad}}=\rho_{\mathrm{end}}\left(\frac{a_{\mathrm{end}}}{a_{\mathrm{rad}}}\right)^{4} \int_{0}^{v_{\mathrm{rad}}}\left(\frac{a(u)}{a_{\mathrm{end}}}\right) e^{-u} d u,
$$

where $v \equiv \Gamma_{\varphi}\left(t-t_{\text {end }}\right)$. Approximating the equation-ofstate parameter as $w \simeq 0$ during reheating, we can further write

$$
\frac{a(t)}{a_{\text {end }}} \simeq\left(\sqrt{\frac{3}{4} \rho_{\text {end }}} \frac{t-t_{\text {end }}}{M_{P}}\right)^{\frac{2}{3}}=\left(\frac{3 H_{\text {end }} v}{2 \Gamma_{\varphi}}\right)^{\frac{2}{3}} .
$$

Substitution of (A9) into (A8) and subsequently into (A6) results in the following simple approximation for the reheating parameter:

$$
\ln R_{\mathrm{rad}} \simeq \frac{1}{6} \ln \left(\frac{\Gamma_{\varphi}}{H_{\mathrm{end}}}\right) .
$$

This result allows us to write simple analytical expressions for the number of $e$-folds after horizon crossing as functions of the effective Yukawa coupling responsible for reheating. As an example for $\alpha=1$, substitution of (A2), (A4), and (A10) into (15) gives

$$
N_{*} \simeq 57.68-\frac{1}{2} \ln N_{*}+\frac{1}{3} \ln y-\frac{1}{12} \ln g_{\mathrm{reh}},
$$

for $\alpha$-Starobinsky models at the pivot scale $k_{*}=$ $0.05 \mathrm{Mpc}^{-1}$ and for T models

$$
N_{*} \simeq 57.82-\frac{1}{2} \ln N_{*}+\frac{1}{3} \ln y-\frac{1}{12} \ln g_{\mathrm{reh}} .
$$

In the range of values shown in the left panels of Fig. 1, the maximum differences of these approximations from the full numerical results are $0.2 \%(0.1 \%)$ for the $\alpha$-Starobinsky models ( $T$ models).

For other analyses of reheating in attractor models, see $[45,53]$.

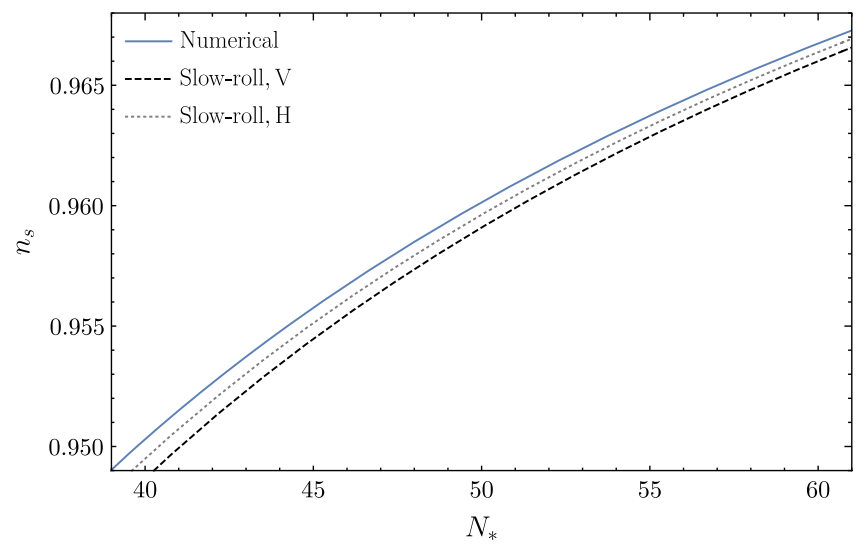

FIG. 3. The scalar tilt $n_{s}$ as a function of the number of $e$-folds after horizon crossing, $N_{*}$, for the $\alpha$-Starobinsky model with $\alpha=1$. The continuous blue line is the numerical solution of Eqs. (B1)-(B4). The dotted gray line is the slow-roll approximation (6) with the Hubble parameters $\epsilon_{H}, \eta_{H}$ defined in (B6). The dashed black line is the slow-roll approximation (6) calculated using the potential parameters $\epsilon, \eta$ defined in (4).

\section{APPENDIX B: COMPUTING THE CMB OBSERVABLES}

In order to compute accurately the inflationary observables, in particular, the scalar tilt $n_{s}$, we have integrated the linear equations for the curvature fluctuation numerically. To calculate the gauge-invariant MukhanovSasaki variable $Q,{ }^{12}$ we integrate the equation of motion [54,55],

$\ddot{Q}+3 H \dot{Q}+\left[\frac{k^{2}}{a^{2}}+3 \dot{\varphi}^{2}-\frac{\dot{\varphi}^{4}}{2 H^{2}}+2 \frac{\dot{\varphi} V_{\varphi}}{H}+V_{\varphi \varphi}\right] Q=0$,

with the Bunch-Davies initial condition $Q_{k \gg a H}=$ $e^{-i k \tau} / a \sqrt{2 k}$, where $d \tau=d t / a$ is the conformal time. The corresponding metric fluctuation and its power spectrum are in turn given by

$$
\begin{gathered}
\mathcal{R}=\frac{H}{|\dot{\varphi}|} Q, \\
\left\langle\mathcal{R}(k) \mathcal{R}^{*}\left(k^{\prime}\right)\right\rangle=\frac{2 \pi^{2}}{k^{3}} \mathcal{P}_{\mathcal{R}} \delta\left(k-k^{\prime}\right) .
\end{gathered}
$$

The scalar tilt is then computed using its definition,

$$
n_{s}=1+\frac{d \ln \mathcal{P}_{\mathcal{R}}}{d \ln k}
$$

and the tensor-to-scalar-ratio is

\footnotetext{
${ }^{12}$ In the Newtonian gauge, $Q=\delta \varphi+\frac{\dot{\varphi}}{H} \Psi$, where $\delta \varphi$ and $\Psi$ denote the field and the metric perturbations, respectively.
} 


$$
r=\frac{\mathcal{P}_{\mathcal{T}}}{\mathcal{P}_{\mathcal{R}}}
$$

where in the case of the tensor spectrum we take the horizon-crossing value $\mathcal{P}_{\mathcal{T}}=2 H^{2} / \pi^{2}$.

Comparing the numerical results obtained by the procedure above with the slow-roll approximations (6) and (7), we find a discrepancy $\gtrsim 1 e$-fold for $N_{*}=N_{*}\left(n_{s}\right)$, see the dashed line in Fig. 3. This difference can be reduced if instead of the potential slow-roll parameters (4) one uses the Hubble slow-roll parameters,

$$
\epsilon_{H}=-\frac{\dot{H}}{H}, \quad \eta_{H}=2 \epsilon_{H}-\frac{\dot{\epsilon_{H}}}{2 \epsilon_{H} H}
$$

see the dotted line in Fig. 3.

This difference remains even when the higher-order slow-roll corrections are included. Ultimately, it is due to the fact that curvature modes do not immediately freeze upon leaving the horizon, which corresponds to the condition $k=a H$. Hence, there is always a shift between the approximate horizon-crossing value, used in our semianalytical estimates, and the final "freeze-out" values used in our full numerical results, in particular, in Fig. 1.
[1] Y. Akrami et al. (Planck Collaboration), Astron. Astrophys. 641, A10 (2020).

[2] A. A. Starobinsky, Phys. Lett. 91B, 99 (1980).

[3] P. A. R. Ade et al. (BICEP and Keck Collaborations), Phys. Rev. Lett. 127, 151301 (2021).

[4] M. Tristram, A. J. Banday, K. M. Górski, R. Keskitalo, C. R. Lawrence, K. J. Andersen, R. B. Barreiro, J. Borrill, L. P. L. Colombo, H. K. Eriksen et al., arXiv:2112.07961.

[5] Y. Akrami et al. (Planck Collaboration), Astron. Astrophys. 643, A42 (2020).

[6] F. L. Bezrukov and M. Shaposhnikov, Phys. Lett. B 659, 703 (2008).

[7] E. Cremmer, S. Ferrara, C. Kounnas, and D. V. Nanopoulos, Phys. Lett. 133B, 61 (1983).

[8] A. B. Lahanas and D. V. Nanopoulos, Phys. Rep. 145, 1 (1987).

[9] J. Ellis, D. V. Nanopoulos, and K. A. Olive, Phys. Rev. Lett. 111, 111301 (2013).

[10] J. Ellis, M. A. G. García, N. Nagata, D. V. Nanopoulos, K. A. Olive, and S. Verner, Int. J. Mod. Phys. D 29, 2030011 (2020).

[11] J. Ellis, D. V. Nanopoulos, and K. A. Olive, J. Cosmol. Astropart. Phys. 10 (2013) 009.

[12] R. Kallosh, A. Linde, and D. Roest, J. High Energy Phys. 11 (2013) 198.

[13] E. Witten, Phys. Lett. 155B, 151 (1985).

[14] R. Kallosh and A. Linde, J. Cosmol. Astropart. Phys. 07 (2013) 002.

[15] R. Kallosh and A. Linde, J. Cosmol. Astropart. Phys. 10 (2013) 033.

[16] J. Ellis, D. V. Nanopoulos, K. A. Olive, and S. Verner, J. Cosmol. Astropart. Phys. 09 (2019) 040.

[17] R. Kallosh and A. Linde, C.R. Phys. 16, 914 (2015).

[18] D. Roest and M. Scalisi, Phys. Rev. D 92, 043525 (2015).

[19] J. R. Ellis, C. Kounnas, and D. V. Nanopoulos, Nucl. Phys. B241, 406 (1984).

[20] R. Kallosh and A. Linde, J. Cosmol. Astropart. Phys. 12 (2021) 008.

[21] J. L. Evans, M. A. G. García, and K. A. Olive, J. Cosmol. Astropart. Phys. 03 (2014) 022.
[22] J. Ellis, D. V. Nanopoulos, K. A. Olive, and S. Verner, J. Cosmol. Astropart. Phys. 08 (2020) 037.

[23] J. Ellis, M. A. G. García, D. V. Nanopoulos, and K. A. Olive, J. Cosmol. Astropart. Phys. 07 (2015) 050.

[24] A. R. Liddle and S. M. Leach, Phys. Rev. D 68, 103503 (2003).

[25] J. Martin and C. Ringeval, Phys. Rev. D 82, 023511 (2010).

[26] N. Aghanim et al. (Planck Collaboration), Astron. Astrophys. 641, A6 (2020); 652, C4(E) (2021).

[27] D. J. Fixsen, Astrophys. J. 707, 916 (2009).

[28] G. F. Giudice, E. W. Kolb, and A. Riotto, Phys. Rev. D 64, 023508 (2001).

[29] J. Ellis, M. A. G. García, D. V. Nanopoulos, K. A. Olive, and M. Peloso, J. Cosmol. Astropart. Phys. 03 (2016) 008.

[30] M. A. G. García, K. Kaneta, Y. Mambrini, and K. A. Olive, Phys. Rev. D 101, 123507 (2020).

[31] C. Pallis, Nucl. Phys. B751, 129 (2006). $<! \longrightarrow$

[32] H. Eberl, I. D. Gialamas, and V. C. Spanos, Phys. Rev. D 103, 075025 (2021).

[33] J. R. Ellis, J. E. Kim, and D. V. Nanopoulos, Phys. Lett. 145B, 181 (1984).

[34] J. R. Ellis, D. V. Nanopoulos, K. A. Olive, and S. J. Rey, Astropart. Phys. 4, 371 (1996).

[35] M. Bolz, A. Brandenburg, and W. Buchmuller, Nucl. Phys. B606, 518 (2001); B790, 336(E) (2008).

[36] S. Kawai and N. Okada, arXiv:2111.03645.

[37] E. Dudas, Y. Mambrini, and K. Olive, Phys. Rev. Lett. 119, 051801 (2017).

[38] M. A. G. García and M. A. Amin, Phys. Rev. D 98, 103504 (2018).

[39] M. Drees and Y. Xu, J. Cosmol. Astropart. Phys. 09 (2021) 012.

[40] J. R. Ellis, D. V. Nanopoulos, K. A. Olive, and K. Tamvakis, Phys. Lett. 118B, 335 (1982); Nucl. Phys. B221, 524 (1983); Phys. Lett. 120B, 331 (1983).

[41] J. Ellis, M. A. G. García, N. Nagata, D. V. Nanopoulos, and K. A. Olive, J. Cosmol. Astropart. Phys. 04 (2019) 009.

[42] M. A. G. García, Y. Mambrini, K. A. Olive, and S. Verner, J. Cosmol. Astropart. Phys. 10 (2021) 061.

[43] M. S. Turner, Phys. Rev. D 28, 1243 (1983). 
[44] M. Drewes, J. Cosmol. Astropart. Phys. 03 (2016) 013.

[45] M. Drewes, J. U. Kang, and U. R. Mun, J. High Energy Phys. 11 (2017) 072.

[46] J. Ellis, M. A. G. Garcia, N. Nagata, D. V. Nanopoulos, and K. A. Olive, J. Cosmol. Astropart. Phys. 01 (2020) 035.

[47] M. Drewes, arXiv:1903.09599.

[48] M. A. G. Garcia, K. Kaneta, Y. Mambrini, K. A. Olive, and S. Verner, arXiv:2109.13280.

[49] D. Maity and P. Saha, J. Cosmol. Astropart. Phys. 07 (2019) 018.

[50] K. D. Lozanov and M. A. Amin, Phys. Rev. D 97, 023533 (2018).
[51] K. Abazajian, G. Addison, P. Adshead, Z. Ahmed, S. W. Allen, D. Alonso, M. Alvarez, A. Anderson, K. S. Arnold, C. Baccigalupi et al., arXiv:1907.04473.

[52] M. Hazumi et al., J. Low Temp. Phys. 194, 443 (2019).

[53] G. German, arXiv:2010.09795.

[54] Z. Lalak, D. Langlois, S. Pokorski, and K. Turzynski, J. Cosmol. Astropart. Phys. 07 (2007) 014.

[55] J. Ellis, M. A. G. García, D. V. Nanopoulos, and K. A. Olive, J. Cosmol. Astropart. Phys. 01 (2015) 010. 